\title{
Altered Neural Responses to Sounds in Primate Primary Auditory Cortex during Slow-Wave Sleep
}

\author{
Elias B. Issa and Xiaoqin Wang \\ Laboratory of Auditory Neurophysiology, Department of Biomedical Engineering, Johns Hopkins University School of Medicine, Baltimore, \\ Maryland 21025
}

How sounds are processed by the brain during sleep is an important question for understanding how we perceive the sensory environment in this unique behavioral state. While human behavioral data have indicated selective impairments of sound processing during sleep, brain imaging and neurophysiology studies have reported that overall neural activity in auditory cortex during sleep is surprisingly similar to that during wakefulness. This responsiveness to external stimuli leaves open the question of how neural responses during sleep differ, if at all, from wakefulness. Using extracellular neural recordings in the primary auditory cortex of naturally sleeping common marmosets, we show that slow-wave sleep (SWS) alters neural responses in the primate auditory cortex in two specific ways. SWS reduced the sensitivity of auditory cortex such that quiet sounds elicited weak responses in SWS compared with wakefulness, while loud sounds evoked similar responses in SWS and wakefulness. Furthermore, SWS reduced the extent of sound-evoked response suppression. This pattern of alterations was not observed during rapid eye movement sleep and could not be easily explained by the presence of slow rhythms in SWS. The alteration of excitatory and inhibitory responses during SWS suggests limitations in auditory processing and provides novel insights for understanding why certain sounds are processed while others are missed during deep sleep.

\section{Introduction}

During sleep, the senses are dulled, yet little is known about underlying auditory cortical function during this state. The loss of conscious awareness in sleep is often compared with the state induced by anesthesia, but this may only be a superficial comparison as evoked potential (Nielsen-Bohlman et al., 1991; Bastuji et al., 2002) and imaging (Portas et al., 2000) studies in humans and, more recently, single neuron recordings in nonhuman primates (Issa and Wang, 2008) have shown a surprising degree of activation in response to sounds in auditory cortex during sleep in a manner that qualitatively resembles that during wakefulness rather than anesthesia. The finding that acoustic information is still reaching auditory cortex during sleep is also inconsistent with the prevailing view that a thalamic gate insulates the cortex from the external sensory environment (Steriade, 2003), as had been suggested by neural recordings across auditory (Edeline et al., 2000), visual (Livingstone and Hubel, 1981), and somatosensory (Mariotti et al., 1989) thalamus during sleep. This raises the possibility of a cortical mechanism such as reduced spiking thresholds or recurrent excitation that increases sensitivity to

\footnotetext{
Received Sept. 19, 2010; revised Nov. 24, 2010; accepted Dec. 26, 2010.

This work was supported by National Institutes of Health Grant DC-03180 (X.W.) and a Whitaker Fellowship (E.B.I.). We thank A. Pistorio, E. Bartlett, D. Bendor, P. Crum, and Y. Zhou for help with animal care; S. Sadagopan for helpful discussion; and Y. Zhou, J. Issa, S. Sadagopan, and C. Miller for comments on an earlier version of this manuscript.

Correspondence should be addressed to Xiaogin Wang, Laboratory of Auditory Neurophysiology, Department of Biomedical Engineering, Johns Hopkins University School of Medicine, 720 Rutland Avenue, Traylor 410, Baltimore, Maryland 21025. E-mail: xiaoqin.wang@jhu.edu.

E. B. Issa's present address: McGovern Institute for Brain Research, Department of Brain and Cognitive Sciences, Massachusetts Institute of Technology, Cambridge, MA 02139.

DOI:10.1523/JNEUROSCI.4920-10.2011

Copyright $\odot 2011$ the authors $\quad 0270-6474 / 11 / 312965-09 \$ 15.00 / 0$
}

thalamic inputs. Steriade and colleagues foresaw the possibility for amplification of cortical responses over thalamic responses in their seminal work on slow oscillations (Steriade et al., 1993, 2001). They suggested that "up" (depolarized) periods of slowbrain rhythms could make neurons unusually excitable during slow-wave sleep (SWS) counteracting or even exceeding the effect of "down" (hyperpolarized) periods (Steriade et al., 2001). Such a mechanism has since been shown to modulate sensory responses in SWS (Massimini et al., 2003) and other nonsleep states (Stern et al., 1997; Poulet and Petersen, 2008). However, it remains unclear how sound-evoked activity in auditory cortex would be modified during SWS given, among other factors, the interplay of slow oscillations and reduced feedforward thalamic input (Hennevin et al., 2007).

Here, we measured auditory cortical responses in SWS across a continuum of sound levels to examine how SWS may modify auditory processing across the dynamic range of hearing. While neurons became less responsive to quiet sounds during SWS compared with wakefulness, they exhibited robust responses at loud sound levels. Furthermore, we observed that sound-evoked suppression was limited in SWS. These observations suggest that acoustic information is not faithfully transferred through auditory cortex in SWS and is instead processed with a limited dynamic range of excitation and inhibition.

\section{Materials and Methods}

Subjects and surgery. Neural data were collected from primary auditory cortex (A1) in four hemispheres of three common marmosets (Callithrix jacchus) (one male, two female) that were also used in our previous study (Issa and Wang, 2008). Animals were adapted to sit in a primate restraint chair for two weeks before a surgery implanting two stainless steel head posts for fixation of the head. Details of implant surgery can be found in 
previous studies (Lu et al., 2001; Wang et al., 2005). After surgery, animals were adapted to experiments during the daytime for at least one month before being switched to nighttime sessions for collection of sleep data. Within the first week of sleep recordings, animals adapted to sleeping in the laboratory in an upright, head-fixed position while sounds were playing. All experimental procedures were approved by the Johns Hopkins University Animal Use and Care Committee.

Physiological recordings. Nightly sessions lasted for 6-8 h (8:00 P.M.-4:00 A.M.). Animals naturally slept in the chair while sounds were played, passing through 5-10 sleep cycles. A $1.1 \mathrm{~mm}$ diameter stainless steel screw was implanted in the caudal temporal lobe for measuring the electroencephalogram (EEG). The EEG was bandpass filtered $(1 \mathrm{~Hz}-500 \mathrm{~Hz})$, amplified $(10,000 \times)$, and displayed on a digital oscilloscope for online monitoring. The EEG and video monitoring (sensitive in the infrared range) of the face and tail were used to assess an animal's behavioral state. Behavioral state was readily identified using standard human sleep scoring techniques (Carskadon and Rechtschaffen, 2000), and details are provided in a previous study (Issa and Wang, 2008). Here, it is important to note that sounds did not awaken the animals even when played at the loudest intensities used in this study. Furthermore, sounds of different intensities were interleaved so that any effect on sleep quality would have affected all sounds equally and been averaged out. Extracellular activity was measured using 2-5 M $\Omega$ tungsten microelectrodes (A-M Systems), bandpass filtered $(300 \mathrm{~Hz}-7 \mathrm{kHz})$, and amplified $(10,000 \times)$. The electrode signal was also bandpass filtered from $1-300 \mathrm{~Hz}$ to obtain the local field potential (LFP). The EEG signal measured from the stainless steel screw was used to assess the behavioral state of the animal, allowing for consistency across sessions. The low frequencies in the LFP signal (recorded from the microelectrode) were used in analyses that compared single neuron activity with ongoing low-frequency rhythms (see Fig. 7). Spike waveforms were sorted online (MSD, Alpha-Omega Engineering) using a template-matching algorithm to identify single units. We regularly obtained well isolated single-units with signal-to-noise ratios $>15$ $\mathrm{dB}$ (median signal-to-noise ratio $=22 \mathrm{~dB}$ ). Neurons were held for an average of $90 \mathrm{~min}$ to allow comparison of neural responses across different states [marmoset sleep cycle $\approx 50 \mathrm{~min}$ (Crofts et al., 2001)]. Details of the identification of Al are given previously (Issa and Wang, 2008).

Acoustic stimuli. Pure tones or narrowband noise were used to drive neurons in A1. In a minority of neurons (5\%), sinusoidal amplitude modulation $(2-128 \mathrm{~Hz})$ was used to achieve more robust drive. The sound level of a preferred stimulus was varied between -10 and $90 \mathrm{~dB}$ sound pressure level (SPL) (although usually in the range of $0-70 \mathrm{~dB}$ SPL) to obtain the intensitytuning curve or rate-level function. Many repeats were generally collected in awake and SWS (median awake $=30$, SWS $=20$ repetitions; minimum required for inclusion in analysis $=10$ repetitions). Stimuli were usually 200 $\mathrm{ms}$ in duration and in some cases $500 \mathrm{~ms}$ long. To limit sleep disruption, presentation rates were generally kept low (1-2 stimuli per second). In analyses examining suppressed responses, we used our complete database of neurons (not just neurons where a rate level was collected). For these data, a larger battery of stimuli was tested as described in our previous work (Issa and Wang, 2008). Experiments were performed in a double-walled sound isolation chamber (Industrial Acoustics) lined with 3 inch acoustic absorption foam (Sonex) to create a soundproof environment. All stimuli were delivered in free-field from a speaker (B\&W) located $0.9 \mathrm{~m}$ directly in front of the animal. The system was calibrated to produce $90 \mathrm{~dB}$ SPL peak output at $1 \mathrm{kHz}$ with little roll-off between 0.2 and $34 \mathrm{kHz}$.
Data analysis. For sound level tuning, a fixed analysis window starting $15 \mathrm{~ms}$ after stimulus onset and ending $75 \mathrm{~ms}$ after stimulus offset was used. Spontaneous firing rates were subtracted from total firing rates such that in all subsequent analyses the terms "rate" or "response" refer to a spontaneous rate-adjusted measure. Responses were considered driven if the mean was $>2.5^{\star}$ SEM above 0 (where SEM was estimated individually for each stimulus). Any rate-level curves with no significant response bins in both awake and SWS were not considered further. Sound level threshold was the quietest sound level eliciting a response $>4{ }^{\star}$ SEM above 0 . In cases where responses did not reach $>4{ }^{\star}$ SEM above 0 , a threshold of $90 \mathrm{~dB}$ SPL was assigned to the nonresponsive state (Fig. $2 B$, denoted as NR). Response latencies were computed using a change point estimation algorithm adapted from Friedman and Priebe (1998). Briefly, two lines of differing slopes were fit to the knee preceding the peak of the response. Latency was defined as the time point at which the slope change was optimal (in the least-squares fit sense). Latencies are only reported for significantly driven responses $\left(>2.5^{\star}\right.$ SEM above 0$)$.

To determine analysis windows for suppressed responses, we adapted an algorithm that we had used previously for detecting driven responses [Issa and Wang (2008); based on the study by Legéndy and Salcman (1985)]. The algorithm starts with a $100 \mathrm{~ms}$ seed window centered at a local minimum that grows in either the left or right direction in $50 \mathrm{~ms}$ segments. Window segments are added as long as their responses are below baseline $\left(p_{\text {soft }}<0.02\right)$, and the response in the whole window meets a more stringent hard criterion ( $\left.p_{\text {hard }}<0.00005\right)$. We also allowed the algorithm to continue as long as the $p$ value decreased toward the hard criterion since $p$ values rarely started below the hard criterion when detecting suppressed events. We assumed Poisson statistics to compute $p$ values from spike counts since low firing rates resulted in non-Gaussian distributions. Even so, responses often deviated from our Poisson assumption because of an unusual number of 0 spike trials, so we performed a post hoc permutation test for the means at the $p<0.01$ level (Rice, 1995). Although the excitatory and inhibitory algorithms were run independently, they usually yielded non-overlapping events (see exam- 
ples in Fig. $3 A-C$ ), which is critical to the assumption that these events represent different processes.

We computed the percentage of modulation of driven responses in sleep for each stimulus by comparing firing rates from two different behavioral states according to the following formulas:

$$
\begin{aligned}
& \% \text { Gain }_{\text {SWS - Awake }}=100 \cdot \frac{r_{\text {SWS }}-r_{\text {Awake }}}{\max \left(\left|r_{\text {SWS }}\right|,\left|r_{\text {Awake }}\right|\right)} ; \\
& \%_{\text {Gain }_{\text {REM - Awake }}}=100 \cdot \frac{r_{\text {REM }}-r_{\text {Awake }}}{\max \left(\left|r_{\text {REM }}\right|,\left|r_{\text {Awake }}\right|\right)},
\end{aligned}
$$

where $r_{\mathrm{SWS}}, r_{\mathrm{REM}}$, and $r_{\text {Awake }}$ are the discharge rates during the SWS, rapid eye movement (REM), and awake conditions, respectively.

For suppressed responses, we computed the percentage of modulation in sleep using an additional measure:

$$
\begin{aligned}
& \%_{\text {Gain }_{\text {SWS }- \text { Awake }}}=100 \cdot\left(\frac{r_{\text {Awake }}}{s_{\text {Awake }}}-\frac{r_{\text {SWS }}}{s_{\text {SWS }}}\right) ; \\
& \% \text { Gain }_{\text {REM }- \text { Awake }}=100 \cdot\left(\frac{r_{\text {Awake }}}{s_{\text {Awake }}}-\frac{r_{\text {REM }}}{s_{\text {REM }}}\right),
\end{aligned}
$$

where $r_{\mathrm{SWS}}, r_{\mathrm{REM}}$, and $r_{\text {Awake }}$ are the discharge rates during suppressed responses, and $s_{\mathrm{SWS}}, s_{\mathrm{REM}}$, and $s_{\text {Awake }}$ are the spontaneous discharge rates during the SWS, REM, and awake conditions, respectively. The ratios in the expressions measure the fraction of spontaneous firing that was suppressed. We used this relative measure to account for the linear dependence of suppression on the baseline firing rate (see Fig. 3D). Normalizing by baseline firing rates removed the scaling relationship between the amount of suppression observed and spontaneous rates (see Fig. 3E).

In general, we report gains for each unit by averaging gains across stimuli. For example, if the unit was responsive to more than one stimulus (e.g., responded to more than one sound level in the quiet range or was suppressed by multiple stimuli), we averaged its gains across these stimuli to obtain an overall unit gain under each condition. The main findings did not change if individual stimuli were used in the analyses instead. If anything, this often increased the statistical power of the results.

For each stimulus trial, the mean EEG amplitude was computed during the stimulus ("Sound" condition) or in the prestimulus period ("No sound" condition). Trials where EEG amplitudes were in the upper quartile were considered up trials while trials where EEG amplitudes were in the lowest quartile were considered down trials (the remaining half of trials were not considered). The modulation of spiking by up versus

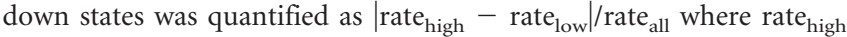
$\left(\right.$ rate $\left._{\text {low }}\right)$ is the mean firing rate in the upper (lower) quartiles of EEG amplitude and rate ${ }_{\text {all }}$ is the mean firing rate across all trials. This trialbased analysis does not depend on the precise timing of spikes and is not based on EEG frequency but simply the mean EEG amplitude in a small window (200-500 ms) either before or during acoustic stimulation. If slow rhythms controlled all variation in spiking, then this measure would approach 1. If slow rhythms had no influence (i.e., little difference in firing rate between low and high EEG epochs), then this measure would approach 0 .

The spike-field coherence (SFC) is a measure of the degree of synchrony between spikes and field potentials in different frequency bands (Fries et al., 2001). The SFC requires the spike-triggered average of the LFPs (STA), which is obtained by aligning and averaging the LFP preceding each spike. A shift predictor was computed by randomly permuting LFP trials and recomputing the STA 10 times. This randomization is important because it removes any stimulus-induced correlations in spikes and LFP activity. Often, in the first 100-200 ms following stimulation, the increase in spike rate and LFP power can artificially induce correlations. Only neurons with STAs that reached absolute values $>2.5^{\star} \mathrm{SD}$ above 0 after shift predictor subtraction were considered further for SFC analysis. The raw SFC is simply the power spectrum of the STA. To distinguish a change in coherence from a change in the strength

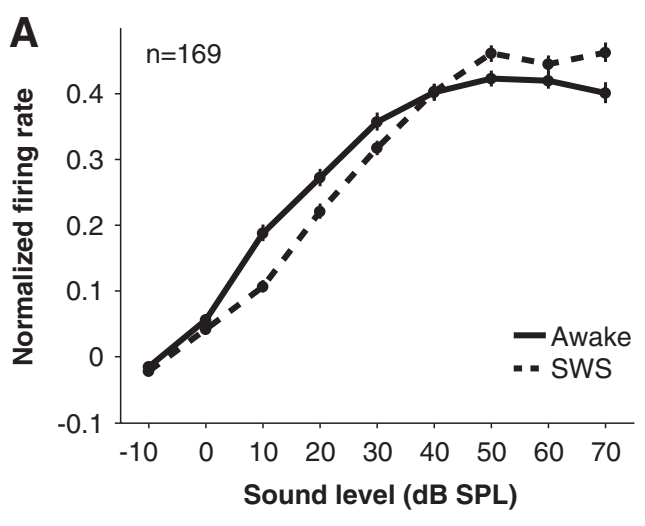

B
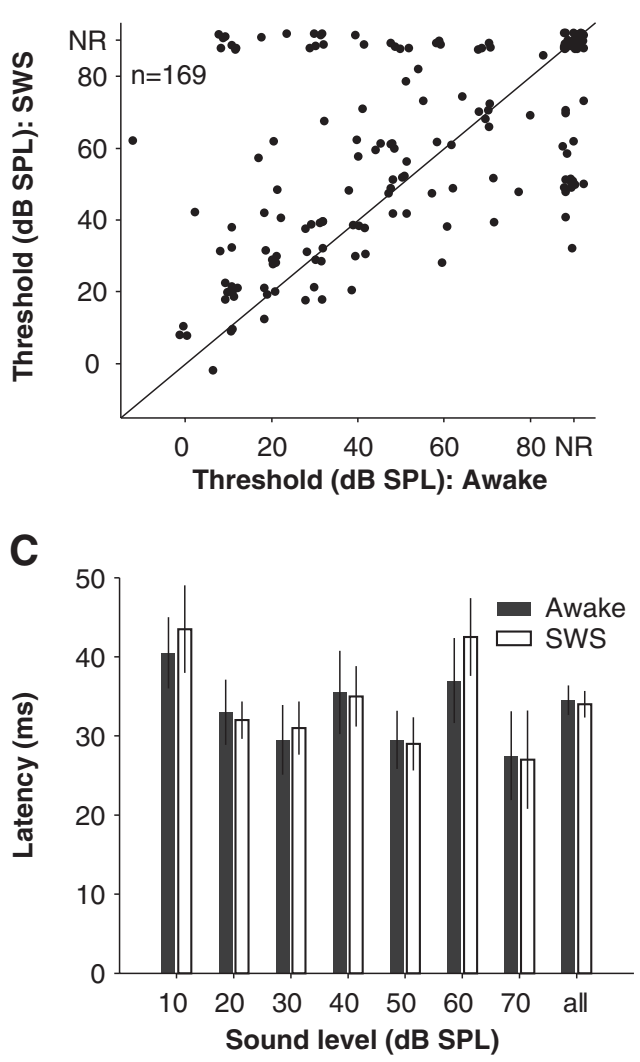

Figure 2. Acoustic thresholds and latencies during SWS. A, Population intensity tuning data in awake (solid) and SWS (dashed). Error bars represent \pm 0.5 SEM. Individual tuning curves for each neuron were first normalized by their peak value in either awake or SWS before being averaged. $\boldsymbol{B}$, Elevated sound level thresholds in SWS compared with wakefulness ( $p=0.01$, Wilcoxon rank sum, $n=169$; NR, not responsive according to a $4 *$ SEM criterion and assigned a threshold of $90 \mathrm{~dB}$ SPL). C, Response latencies in SWS (open bars) were similar to those in wakefulness (filled bars) across all sound levels tested (median latency awake $=34.5+1.9$ vs SWS $=34.0+1.7 \mathrm{~ms}, p=0.71$, Wilcoxon rank sum, $n=244$, "all" condition).

of oscillations in the LFP, the raw SFC was normalized by the mean spectrum of all LFPs used in calculating the STA. This normalization step is important since the LFP during SWS is higher in amplitude than during awake and REM.

\section{Results}

Sound level dependence of neural responses in slow-wave sleep

Sound level is an important parameter for determining when a sound becomes salient during sleep. We measured the rate-level functions of 169 neurons in A1 of three naturally sleeping mar- 
moset monkeys during at least one episode of SWS and one episode of wakefulness. A range of sound levels was tested (usually 0-70 dB SPL) without awakening the animal. A representative neuron is shown in Figure $1 A$. In the awake condition, this neuron began to respond to a $780 \mathrm{~Hz}$ pure tone at $20 \mathrm{~dB}$ SPL, but its response to the same tone disappeared at 20 and $30 \mathrm{~dB}$ SPL during SWS (Fig. $1 A$, middle plot, black arrows), even though the responses at louder sound levels were preserved.

In our sample of 169 A1 neurons, 69 neurons were responsive $\left(>2.5^{\star} \mathrm{SEM}\right.$ above baseline) to quiet sounds $(0-20 \mathrm{~dB})$ and 106 neurons were responsive to loud sounds (70-90 dB). Firing rates during wakefulness were similar in these sound level regimes (mean firing rates quiet $=$ $13.5 \pm 1.1$ vs loud $=14.9 \pm 1.3$ spikes s $^{-1}$, $p=0.78$, Wilcoxon rank sum, $n_{\text {quiet }}=69$, $\left.n_{\text {loud }}=106\right)$, consistent with the nonmonotonic sound level tuning commonly seen in A1 of unanesthetized animals (Brugge and Merzenich, 1973; Pfingst and O'Connor, 1981; Sadagopan and Wang, 2008). During SWS, however, the population of neurons showed a large drop in activity for quiet sounds (gain $=-33 \pm$ $6 \%$ ) but not for loud sounds (gain $=9 \pm$ $5 \%)\left(p<0.01\right.$, Wilcoxon rank sum, $n_{\text {quiet }}=$ $\left.69, n_{\text {loud }}=106\right)($ Fig. $1 B)$. The gain distribution for quiet sounds was shifted toward negative gains ( $p<0.01, t$ test, $n=$ $69)$ implying that responses during SWS were significantly weaker than those during wakefulness (Fig. 1B, green curve). For louder sound levels, however, the gain distribution was not significantly shifted from zero ( $p=0.08$, $t$ test, $n=106$ ), indicating similar responses between wakefulness and SWS (Fig. $1 \mathrm{~B}$, black curve). As a result, overall firing rates for quiet sounds in SWS were $~ 35 \%$ weaker compared with wakefulness (0-20 dB SPL: awake $=10.7 \pm 1.1$ vs SWS $=7.0 \pm 0.9$ spikes $\mathrm{s}^{-1}, p<0.01$, Wilcoxon rank sum, $n=69$ ) (Fig. $1 B$, inset, left bars) while firing rates were not significantly different between the awake and SWS conditions for loud sounds ( $p=0.47$, Wilcoxon rank sum, $n=106$ ) (Fig. $1 B$, inset, right bars). This phenomenon is further illustrated by the population average sound level function (Fig. 2A), which shows that the average awake response is greater than the average SWS response at low sound levels. This trend was reversed at high sound levels. The decrease in sensitivity to quiet sounds resulted in elevated acoustic thresholds during SWS (mean shift $=8 \pm 2 \mathrm{~dB}$, $p=0.01$, Wilcoxon rank sum, $n=169$ ) (Fig. $2 B$ ). One plausible mechanism for elevating acoustic thresholds in SWS is elevated spiking thresholds. However, as mentioned above, firing rates for quiet sounds were similar to those for loud sounds suggesting that these
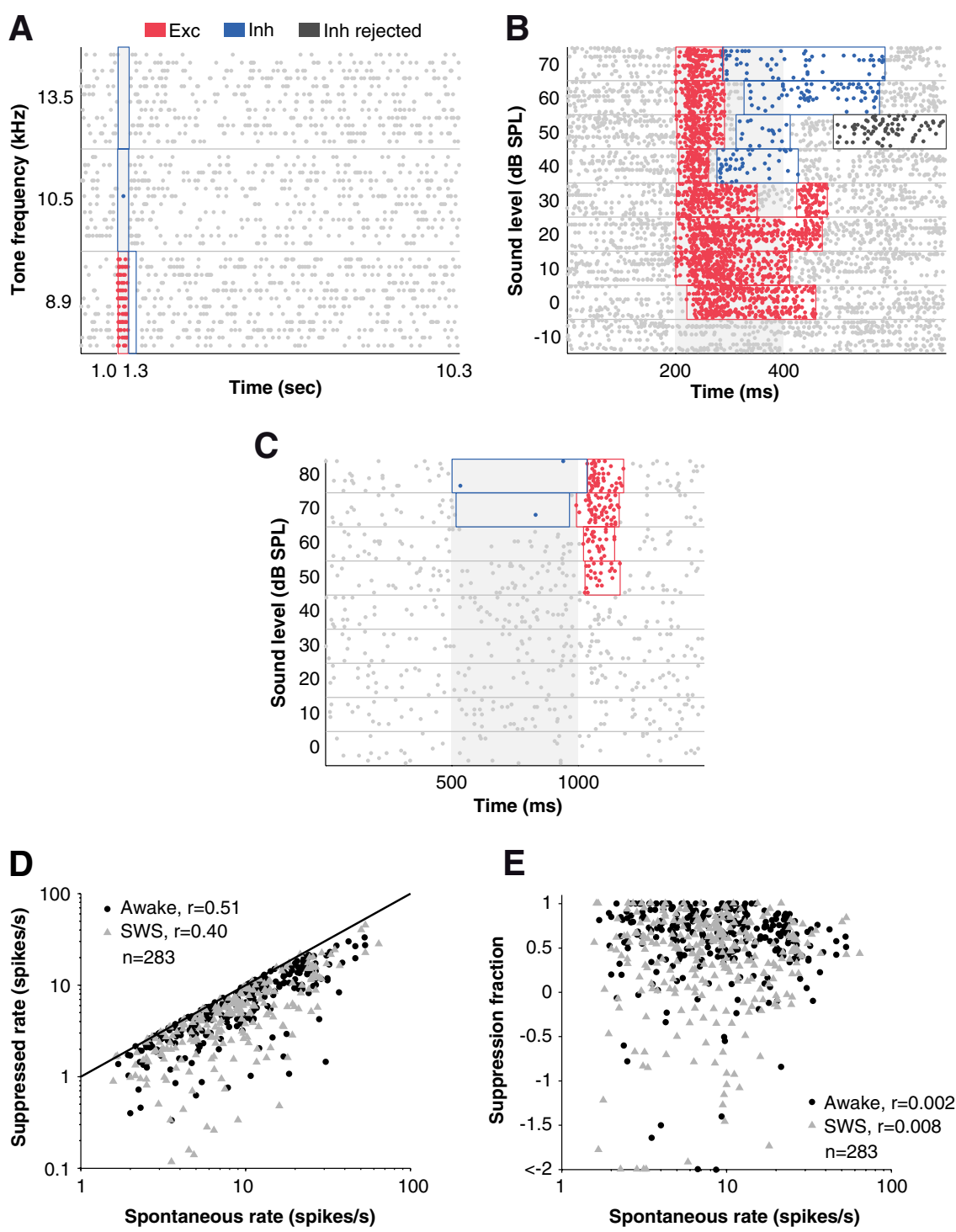

Figure 3. Detection of suppressed events and dependence on spontaneous rate. $\boldsymbol{A}$, Well defined periods of excitation (Exc; red) and inhibition (Inh; blue) were detected among a background of high spontaneous firing by the windowing algorithm (see Materials and Methods). No false windows were detected in the 9-s-long period of spontaneous firing following the stimulus. $\boldsymbol{B}$ The varying durations of excitatory responses were well captured by the algorithm. At higher sound levels, excitation was shorter in duration and was followed by inhibition. In some cases, an inhibitory window was detected by the algorithm but then thrown out (dark gray) because it did not pass a post hoc permutation test for the means when compared with spontaneous firing. C, An example where inhibition preceded excitation at high sound levels. Note that windows were non-overlapping even though excitatory and inhibitory algorithms were run independently. $\boldsymbol{D}$, Suppressed rates which were already spontaneous-rate subtracted were still highly correlated with spontaneous rates $(r=0.45, p<0.01$, Pearson's correlation coefficient, $n=566)$, suggesting a scaling relationship between spontaneous rate and suppression. At higher spontaneous rates, higher suppression can be observed extracellularly. This was not a ceiling effect where spontaneous rates limited the amount of suppression that could be observed extracellularly since in most cases firing rates were not suppressed to 0 (i.e., suppression = spontaneous; unity line). $\boldsymbol{E}$, For the analyses of Figures $4 B$ and $6 B$ in Results, suppressed rates were normalized by spontaneous rate to obtain the fraction of spontaneous rate that was suppressed. This measure removed correlation with spontaneous rate $(r=0.002, p=0.26$, Pearson's correlation coefficient, $n=566$ ).

stimuli were equally above neural firing thresholds. Second, we examined response latencies as a possible correlate of spiking thresholds and found that response latencies were almost identical in wakefulness and SWS regardless of sound level ( $p=0.71$, Wilcoxon rank sum, $n=244$ stimuli) (Fig. $2 C$ ). These results suggest that a change in sound-response threshold (i.e., the lack of responsiveness to quiet sounds in SWS) may not be directly related to spiking threshold (i.e., the relationship between spiking and membrane potential). 
A

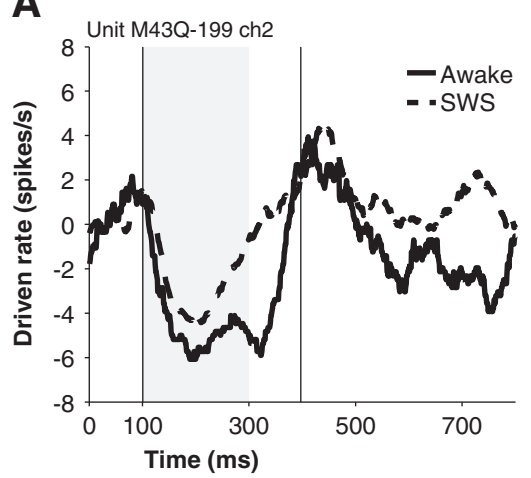

B

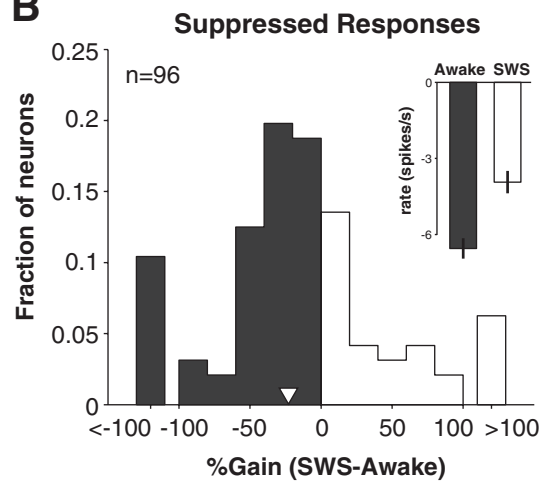

Figure 4. Suppressed responses during wakefulness and SWS. A, An example neuron whose response was suppressed during presentation of a $20 \mathrm{kHz}$ pure tone $(70 \mathrm{~dB} \mathrm{SPL}$ ) and for a short time following (vertical lines represent analysis window returned by windowing algorithm, see Materials and Methods). Suppression was weaker and shorter-lasting in SWS (dashed) than in awake (solid) (gain $=-24 \%$ ). Curves were generated by first subtracting spontaneous rates and then smoothing with a 50 ms moving average window. $\boldsymbol{B}$, Percentage change of the strength of suppression in SWS. The distribution is shifted toward negative gains (mean $=-23 \%$, white inverted triangle) indicating that responses were not suppressed as strongly in SWS as in wakefulness. Inset, Population-averaged firing during suppression events did not go as far below baseline firing ( 0 in this figure) during SWS as during wakefulness.

A

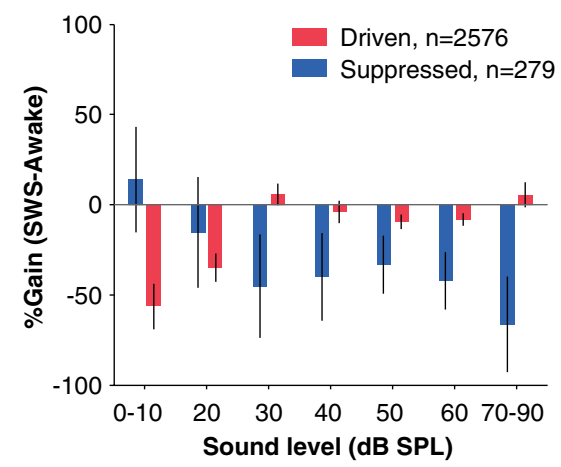

B

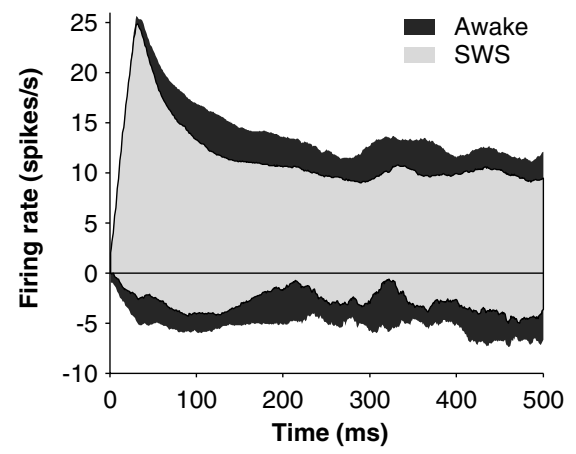

Figure 5. Population-averaged activity during wakefulness and SWS. A, Changes in population-averaged driven and suppressed responses as a function of sound level. Suppressed responses were weakened in SWS across a wide range of sound levels (negative blue bars). However, driven responses were weakened only at extremely quiet sound levels (negative red bars). Population-averaged activity was obtained by summing firing rates of all detected driven or suppressed events at each sound level without normalizing and then computing the percentage gain between awake and SWS. Error bars represent \pm 1 SEM. $\boldsymbol{B}$, Population-averaged histogram of all driven (positive) and suppressed (negative) responses showing their time course. Because SWS responses (light gray) were not driven or suppressed as strongly as in awake (dark gray), the dynamic range of SWS was limited (light shaded area is encompassed by dark area). Population poststimulus time histograms were obtained by averaging all detected driven $(n=3133)$ and suppressed events $(n=272)$ during the time window in which they occurred without normalizing. Responses were smoothed by a $30 \mathrm{~ms}$ moving average window.

\section{Suppressed responses during acoustic stimulation}

An important property in sharpening neural tuning (Wu et al., 2008; Sadagopan and Wang, 2010) and expanding dynamic range (Wu et al., 2006; Tan et al., 2007) is inhibition. Here, we isolated events where firing rate was suppressed below spontaneous firing rate (see Materials and Methods) as a possible window into inhibitory processes (Fig. 3A-C). In general, detecting inhibition extracellularly depended on background firing rate such that neurons with higher background firing rates exhibited stronger suppression (Fig. 3D). We normalized for this dependency by rescaling firing rates (i.e., fractional suppression $=$ suppressed rate/spontaneous rate) (Fig. $3 E$ ). This normalized measure allowed comparison of inhibition across behavioral states independent of changes in baseline firing rate.

Figure $4 A$ shows the time course of suppressed responses in an example neuron during the awake and SWS conditions. The neu- ron was more suppressed during wakefulness $(\sim 24 \%)$ than during SWS. Over the population of neurons in Al showing a suppressed response, the average gain of suppressed events was $-23 \pm 10 \%$ (negative gain implies that suppression was stronger in wakefulness compared with SWS) (Fig. $4 B$, white inverted triangle) and approached significance $(p=0.03, t$ test, $n=96$ ). This resulted in a $40 \%$ change in population averaged firing rate during suppressed responses (median firing rate below baseline: awake $=$ $-6.5 \pm 0.4$ vs SWS $=-3.9 \pm 0.4$ spikes $\mathrm{s}^{-1}, p<0.01$, Wilcoxon rank sum, $n=$ 283) (Fig. $4 B$, inset). Changes in spontaneous firing were not likely causing this effect as spontaneous firing rates did not significantly differ between the awake and SWS conditions (mean spontaneous rate: awake $=9.9 \pm 0.9$ vs SWS $=9.7 \pm 0.9$ spikes $\mathrm{s}^{-1}, p=0.89$, Wilcoxon rank sum, $n=96)$. If we used absolute firing rates (not normalized by spontaneous rate), we obtained similar results $($ gain $=-22 \pm 8 \%, p=0.006, t$ test, $n=96$ ). Also, modulation of spontaneous rates was generally smaller than the magnitude of change in suppressed rates (mean $\mid \Delta$ suppressed $\mid=7.2 \pm 0.7$ vs $\mid \Delta$ spontaneous $\mid=4.4 \pm 0.5$ spikes $\left.\mathrm{s}^{-1}, p<0.01, n=96\right)$.

From the data presented in Figures 1 and 4, SWS modulation of neural activity was in the form of a loss of driven responses above spontaneous activity and a loss of suppression below spontaneous activity. Driven and suppressed activity levels are summarized for the population as a function of sound level in Figure $5 A$. The decrease in driven responses (red bars) and suppressed responses (blue bars) limited the range of possible responses in SWS by $\sim 40 \%$. This suggests that there is a smaller dynamic range of responses during SWS compared with wakefulness, which may place constraints on the amount of information that can be transferred across sound levels by A1 neurons. This limitation in dynamic range was present throughout the time course of the population response, especially during the sustained portion of the response (Fig. 5B).

\section{Neural responses during rapid eye movement sleep}

The changes observed in Al during SWS were not as prominent in REM sleep. As shown in Figure 6A, sleep-wakefulness gains for quiet sounds were only weakly negative (REM response slightly weaker than awake response) (mean gain $_{0-20}=-4+6 \%$ ) and were similar to gains for loud sounds (mean gain ( $_{70-90}=-13+$ $5 \%, p=0.58$, Wilcoxon rank sum, $n_{0-20}=67, n_{70-90}=90$ ). Overall, mean firing rates for quiet sounds were slightly but not significantly reduced ( $p=0.35$, Wilcoxon rank sum, $n=67$ ) (Fig. 6A, inset, left bars). Furthermore, neurons in REM did not 
show a loss of suppressed responses compared with wakefulness (mean gain = $-0.3 \pm 7.1 \%, p=0.96, t$ test, $n=99$ ) (Fig. $6 B$ and inset).

\section{Influence of slow rhythms on neural} responses to sounds during sleep Ongoing slow brain rhythms $(<5 \mathrm{~Hz})$ are known to modulate neural firing in awake, anesthetized, and asleep states (Steriade et al., 1993, 2001; Le Van Quyen et al., 2010), and there is evidence that this coupling extends to the theta range (4-8 $\mathrm{Hz}$ ) (Canolty et al., 2006), but it is unclear how this modulation of neural firing in auditory cortex would be affected by the presence of acoustic stimuli during SWS. In one extreme scenario, neural activity would be dominated by EEG up and down states. At another extreme, external sounds may override slow rhythms. An intermediate possibility is that slow rhythms modulate existing responses to external sounds (e.g., multiplicative gain). To address this issue, we analyzed the difference in firing rates between EEG up states and down states (see Materials and Methods). In the example neuron shown in Figure 7A, the background firing rate (i.e., no external stimulation) increased during up states (depolarized EEG) in SWS and decreased during down states (hyperpolarized EEG) in SWS (up $=24.4 \pm 10.3$ vs down $=10.6 \pm 3.7$ spikes s ${ }^{-1}, p<0.01$, Wilcoxon rank sum, $n=$ 9) (Fig. $7 A$, left bars). This modulation with EEG amplitude, however, was absent during acoustic stimulation in this example neuron; firing rates were no longer significantly different between the two conditions $(\mathrm{up}=60.1 \pm 7.3$ vs down $=63.4 \pm 4.8$, $p=0.53$, Wilcoxon rank sum, $n=9$; spontaneous firing rate not subtracted) (Fig. $7 A$, right bars). In our population of neurons, modulation by slow rhythms during acoustic stimulation was weaker than during spontaneous activity in all behavioral states (awake: no sound $=53 \pm 6 \%$ vs sound $=27 \pm 2 \%, p<0.01$, Wilcoxon rank sum, $n=75$; SWS: no sound $=68 \pm 6 \%$ vs sound $=26 \pm 2 \%, p<0.01$, Wilcoxon rank sum, $n=96$; REM: no sound $=32 \pm 4 \%$ vs sound $=21 \pm 2 \%, p=0.10$, Wilcoxon rank sum, $n=78$ ) (Fig. $7 B$, no sound vs sound condition). Note that if the effects of slow rhythms were purely multiplicative, then fractional modulation of firing rates should have been similar between spontaneous and acoustically driven periods of activity. Rather, EEG modulation of responses in SWS was greatly reduced during acoustic stimulation and became similar to levels measured in wakefulness $\left[p=0.85\right.$, Wilcoxon rank sum, $n_{\mathrm{SwS}}=$ $\left.96, n_{\text {Awake }}=75\right)($ Fig. $7 B$, sound condition; SWS (gray) vs awake (black)].

Although slow rhythms had a weak influence on spike rate during acoustic stimulation, they could influence spike timing independently of changes in spike rate. For example, the same number of spikes could become more tightly locked to lowfrequency rhythms during SWS. We used the SFC to measure locking of spikes to the EEG (Fries et al., 2001). We found that locking to slow rhythms was most enhanced in SWS as compared with wakefulness (SFC at $1 \mathrm{~Hz}$ : awake $=3.4^{\star} 10^{-3} \pm 0.3^{\star} 10^{-3}$ vs SWS $=4.4^{\star} 10^{-3} \pm 0.3^{\star} 10^{-3} \mathrm{mV}^{2}, p<0.01$, Wilcoxon rank sum, $n=258$ ) and REM (SFC at $1 \mathrm{~Hz}$ : REM $=3.7^{\star} 10^{-3} \pm$ $0.4^{\star} 10^{-3}$ vs SWS $=4.4^{\star} 10^{-3} \pm 0.3^{\star} 10^{-3} \mathrm{mV}^{2}, p<0.01$, Wilcoxon rank sum, $n=258$ ) (Fig. $7 C$, solid lines). This strong coherence during SWS, however, declined dramatically with acoustic stimulation (SFC at $1 \mathrm{~Hz}$ SWS: spontaneous = $4.4^{\star} 10^{-3} \pm 0.3^{\star} 10^{-3}$ vs acoustically driven $=1.1^{\star} 10^{-3} \pm$ $0.2^{\star} 10^{-3} \mathrm{mV}^{2}, p<0.01$, Wilcoxon rank sum, $n=258$ ) (Fig. $7 C$, compare solid to dashed gray line). It is important to note that the SFC is corrected for any locking induced by the stimulus and for differences in overall EEG power between states (see Materials and Methods).

The results in Figure 7, $B$ and $C$, suggest a limited role of slow rhythms in influencing neural responses during acoustic stimulation. Of interest to this study, however, is whether the influence of slow rhythms differed between quiet and loud sound levels. A differential effect could help explain our finding that only responses to quiet sounds were weakened in SWS (Fig. 1). We found, however, that the influence of slow rhythms was similar for quiet and loud sounds whether in modulating spike rate ( $p=$ 0.76 , Wilcoxon rank sum, $\left.n_{\text {quiet }}=25, n_{\text {loud }}=40\right)($ Fig. $7 D)$ or spike timing ( $p=0.20$, Wilcoxon rank sum, $n=169)$ (Fig. $7 E$ ). In summary, slow rhythms had a much weaker effect on externally driven activity than on spontaneous activity, and these effects were just as weak for quiet and loud sounds. Therefore, slow rhythms are unlikely to account for the differential loss of responses to quiet sounds in SWS.

\section{Discussion}

In the present study, we have demonstrated how sleep alters cortical responses to sounds compared with wakefulness. These alterations, however, were not revealed by all sounds but were specific to quiet sounds and sounds that suppressed neural responses. Previous work did not reveal these effects of SWS because sounds were typically played at moderate to high sound levels intended to drive neurons in an above threshold regime, and suppressed responses were not analyzed (Peña et al., 1999; Edeline et al., 2001). It remains to be seen what possible mechanisms may account for the decreased sensitivity of neurons during SWS. Ideally, this would require detailed intracellular recordings that are difficult to accomplish across sleep-wake transitions. Alternatively, principled manipulation of more complex stimuli and further characterization of neural receptive fields during SWS could provide insights into how neurons are modulated. Our results suggest, however, a limited role for slow 
A

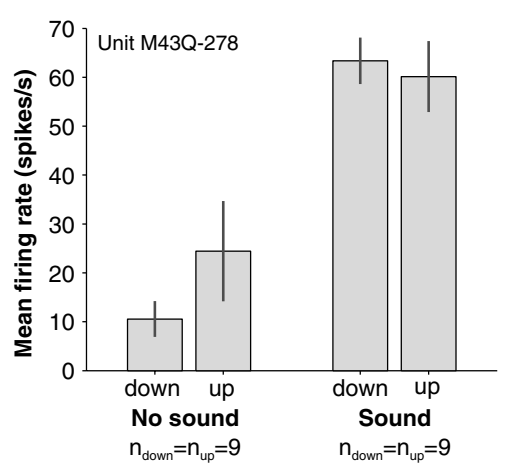

C

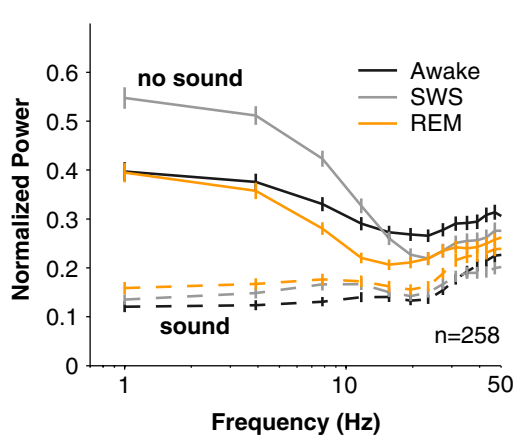

E

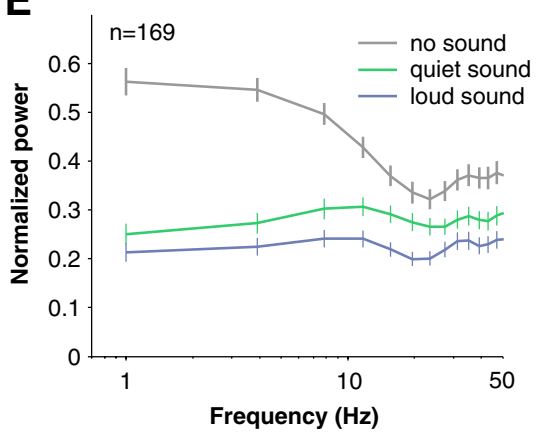

Figure 7. Influence of slow rhythms on neural activity in awake, SWS, and REM. $\boldsymbol{A}$, An example neuron whose background activity showed EEG state-dependent firing rate modulation (left bars). Trials $(n=35)$ were divided into quartiles based on EEG amplitude. Trials in the lower quartile $(n=9)$ and the upper quartile $(n=9)$ were compared for effects of firing rate modulation with EEG amplitude. During EEG down states, this example neuron's firing rate was reduced by more than half. Upon acoustic stimulation with a $11.9 \mathrm{kHz}$ pure tone (70 $\mathrm{dB} S \mathrm{SP}$ ), there was no longer a difference in total firing rates between up and down EEG states (right bars, spontaneous firing rate not subtracted). Error bars represent \pm 1 SEM. B, EEG amplitude modulated spiking especially when no sounds were playing in SWS (gray bar, no sound condition). Effects were generally stronger in SWS than in REM (orange) or wakefulness (black). During acoustic stimulation, however, modulation of spike rate by EEG amplitude became much weaker compared with the activity evoked by sounds (compare no sound to sound conditions; SWS: no sound $=68 \pm 6 \%$ vs sound $=26 \pm 2 \%, p<0.01$, Wilcoxon rank sum, $n=96$ ) and slow rhythm modulation no longer differed between SWS and wakefulness (sound condition: $S W S=26+2 \%$ vs awake $=27+2 \%, p=0.85$, Wilcoxon rank sum, $n_{\text {sws }}=96, n_{\text {Awake }}=75$ ). Only neurons with spontaneous rates $>3$ spikes s ${ }^{-1}$ were included in this analysis so that appreciable variability occurred in trial firing rates. Error bars represent \pm 1 SEM. C, Locking of spontaneous activity to low-frequency EEG rhythms was enhanced in SWS compared with wakefulness (solid gray and black lines). This cannot be simply explained by the stronger power in the low-frequency EEG in SWS since the spike-field coherence measure normalizes for differences in EEG power across the different behavioral states (see Materials and Methods). In general, locking to low-frequency rhythms was poor while sounds were being played (dashed lines). Error bars represent \pm 1 SEM. $D$, High (top quartile of EEG amplitudes) and low (bottom quartile) trials could cause a $75 \pm$ $9 \%$ swing in firing rates as seen in the overall modulation in spontaneous firing rate between up and down periods (black bar). This modulation with EEG amplitude, however, was reduced to $27+3 \%$ and $23+3 \%$ during playing of quiet (green, $0-20 \mathrm{~dB}$ ) and loud (blue, $70-90 \mathrm{~dB})$ sounds respectively $\left(p_{\text {(no sound vs quiet sound) }}=1.4^{*} 10^{-5}, p_{(\text {no sound vs loud sound })}=5.5^{*} 10^{-6}\right.$, Wilcoxon rank sum, $n_{\text {no sound }}=$ $51, n_{\text {quiet }}=25, n_{\text {loud }}=40$ ). Only neurons with spontaneous rates $>3$ spikes $s^{-1}$ were used in this analysis. Otherwise, iffiring rates were toolow, little trial-by-trial variation could be observed. Error bars represent \pm 1 SEM. $E$, TheSFC was strongest for low frequencies indicating that spikes were locked to slow brain rhythms. This locking was strongly diminished whether quiet $(0-20 \mathrm{~dB})$ or loud ( $70-90 \mathrm{~dB})$ sounds were played (SFC at $1 \mathrm{~Hz}$ : no sound $=4.3^{*} 10^{-3}+0.4^{*} 10^{-3}$ vs quiet sound $=2.1^{*} 10^{-3}+0.3^{*} 10^{-3} \mathrm{mV}^{2}$ vs loud sound $=$ $1.8^{*} 10^{-3}+0.4^{*} 10^{-3} \mathrm{mV}^{2}, p_{\text {(no sound vs quiet sound) }}<0.01, p_{\text {(no sound vs loud sound) }}<0.01$, Wilcoxon rank sum, $n=169$ ). Error bars represent \pm 1 SEM. rhythms across sound level. The weakened influence of up and down states during quiet levels of acoustic stimulation $<30 \mathrm{~dB}$ SPL suggests that other mechanisms are involved in addition to slow oscillations and that sleep is a complex interplay of many factors at both the cellular and network levels.

\section{Possible mechanisms}

A simple mechanism for increasing soundresponse thresholds would be to increase neural spiking thresholds (i.e., the relationship between membrane potential and spiking). However, we have found that response gains between wakefulness and SWS are similar across a range of firing rates, even near threshold (Issa and Wang, 2008). And in the present study, we report that response latencies in SWS are as short as those during wakefulness. These extracellular data cannot rule out changes in intracellular thresholds during SWS but suggest that the relationship between spiking thresholds and acoustic thresholds may not be straightforward.

Two salient properties of SWS are a reduced thalamic drive (Livingstone et al., 1981; Mariotti et al., 1989; Edeline et al., 2000) and prominent low-frequency oscillations (Steriade et al., 1993, 2001; Le Van Quyen et al., 2010). We did indeed observe a role of slow rhythms in modulating background firing. This modulation was not as strong, however, as modulation by external stimuli. This is not surprising especially since we chose stimuli intended to drive neurons well. We note here that our data only extended down to $1 \mathrm{~Hz}$, whereas slow oscillations, as originally described, are in the $0.1-1 \mathrm{~Hz}$ range (Steriade et al., 1993), so it is still possible that EEG influences may be found in this low range. But behavior in the 1-5 $\mathrm{Hz}$ range can be an indicator of the influences of up and down periods as the SFC in this range was much stronger in SWS than in awake and REM (Fig. 7C), suggesting the involvement of a broadly defined low-frequency range in SWS (Stern et al., 1997; Destexhe et al., 1999; Lampl et al., 1999). Little data exist on the relative influence of slow oscillations and external stimuli on neural responses during natural sleep. One study in sleeping humans reported clear changes in somatosensory evoked potentials with the phase of slow oscillations (Massimini et al., 2003), but further studies will be needed especially at the neural level in auditory cortex to reveal the underlying dynamics.

Given that thalamic responses decrease during SWS, it seems plausible that this would lead to less bottom-up excita- 
tion and inhibition in cortex. This view, simple as it may be, explains our dual observations of weakened drive and suppression. If the change in excitatory and inhibitory processes is balanced, then there would be no net change in overall firing rates. This is consistent with the observation that overall activity is preserved from wakefulness to SWS in cortex (Issa and Wang, 2008). The properties that we observed in cortex could be directly inherited from thalamus, but a study in the auditory thalamus comparing rate-level and frequency tuning between SWS and wakefulness found uniform gains (consistently depressed thalamic responses in SWS) across sound level and frequency (Edeline et al., 2000), suggesting that our observations in cortex are unique to the thalamocortical transformation and not to earlier stages of processing.

A key prediction from our data is that inhibitory processes are weakened during SWS. This hypothesis would be easy to test either directly through intracellular recordings or indirectly by observing signatures of inhibition, such as its noted presence at high sound levels (Wu et al., 2006; Tan et al., 2007; Sadagopan and Wang, 2010) or its influence on correlated network activity (Wang, 2010). We did not test sound levels beyond $70 \mathrm{~dB}$ SPL except in a few cases to limit awakening the animals. But our data suggest a weak trend for SWS responses to overtake awake responses had we tested further $>70 \mathrm{~dB}$ SPL. In this regime, inhibition is thought to play an increasing role.

\section{Functional implications}

It is difficult to speculate how neural responses in auditory cortex will translate into perception of sounds during sleep, but our data provide constraints on the processing possible during SWS and complement human behavioral and functional imaging work. The elevated acoustic thresholds that we observed at the neural level are consistent with elevated human arousal thresholds during SWS (Bonnet, 1982). Furthermore, preserved overall activity levels during SWS may still allow for residual discrimination of salient or behaviorally important acoustic events as observed in sleeping humans who will respond to their name being called (Oswald et al., 1960; Bastuji et al., 2002) or eventually awaken to the sound of an alarm clock.

Functional imaging in asleep and lightly sedated patients has suggested that auditory cortex is active but that processing in higher level areas may be compromised (Davis et al., 2007). Evoked potential studies have also found changes in SWS for higher level processing (Cote, 2002). It is possible that deficits in coding fidelity in primary auditory cortex during SWS may lead to deteriorated read-out in higher areas and limited discrimination at the behavioral level. One prediction of our data is that neurons should not be able to respond as dynamically to time-varying features of sounds since the range for analog coding with firing rate is reduced during SWS (Fig. 5). This is speculative and only highlights the importance of recording from higher areas during sleep to bridge to perception (Issa and Wang, 2008). Future work along these lines will help elucidate the mechanisms of auditory processing during sleep and provide neural insight into how most sounds escape our conscious awareness during sleep.

\section{References}

Bastuji H, Perrin F, Garcia-Larrea L (2002) Semantic analysis of auditory input during sleep: studies with event related potentials. Int J Psychophysiol 46:243-255.

Bonnet MH (1982) Performance during sleep. In: Biological rhythms, sleep and performance (Webb WB, ed), pp 205-237. Chichester, UK: Wiley.

Brugge JF, Merzenich MM (1973) Responses of neurons in auditory cortex of the macaque monkey to monaural and binaural stimulation. J Neurophysiol 36:1138-1158.

Canolty RT, Edwards E, Dalal SS, Soltani M, Nagarajan SS, Kirsch HE, Berger MS, Barbaro NM, Knight RT (2006) High gamma power is phase-locked to theta oscillations in human neocortex. Science 313:1626-1628.

Carskadon MA, Rechtschaffen A (2000) Monitoring and staging human sleep. In: Principles and practices of sleep medicine, Ed 3 (Kryger MH, Roth T, Dement WC, eds), pp 1197-1215. Philadelphia: W.B. Saunders.

Cote KA (2002) Probing awareness during sleep with the auditory odd-ball paradigm. Int J Psychophysiol 46:227-241.

Crofts HS, Wilson S, Muggleton NG, Nutt DJ, Scott EA, Pearce PC (2001) Investigation of the sleep electrocorticogram of the common marmoset (Callithrix jacchus) using radiotelemetry. Clin Neurophysiol 112:2265-2273.

Davis MH, Coleman MR, Absalom AR, Rodd JM, Johnsrude IS, Matta BF, Owen AM, Menon DK (2007) Dissociating speech perception and comprehension at reduced levels of awareness. Proc Natl Acad Sci U S A 104:16032-16037.

Destexhe A, Contreras D, Steriade M (1999) Spatiotemporal analysis of local field potentials and unit discharges in cat cerebral cortex during natural wake and sleep states. J Neurosci 19:4595-4608.

Edeline JM, Manunta Y, Hennevin E (2000) Auditory thalamus neurons during sleep: changes in frequency selectivity, threshold, and receptive field size. J Neurophysiol 84:934-952.

Edeline JM, Dutrieux G, Manunta Y, Hennevin E (2001) Diversity of receptive field changes in auditory cortex during natural sleep. Eur J Neurosci 14:1865-1880.

Friedman HS, Priebe CE (1998) Estimating stimulus response latency. J Neurosci Methods 83:185-194.

Fries P, Reynolds JH, Rorie AE, Desimone R (2001) Modulation of oscillatory neuronal synchronization by selective visual attention. Science 291:1560-1563.

Hennevin E, Huetz C, Edeline JM (2007) Neural representations during sleep: from sensory processing to memory traces. Neurobiol Learn Mem $87: 416-440$.

Issa EB, Wang X (2008) Sensory responses during sleep in primate primary and secondary auditory cortex. J Neurosci 28:14467-14480.

Lampl I, Reichova I, Ferster D (1999) Synchronous membrane potential fluctuations in neurons of the cat visual cortex. Neuron 22:361-374.

Le Van Quyen M, Staba R, Bragin A, Dickson C, Valderrama M, Fried I, Engel J (2010) Large-scale microelectrode recordings of high-frequency gamma oscillations in human cortex during sleep. J Neurosci 30:7770-7782.

Legéndy CR, Salcman M (1985) Bursts and recurrences of bursts in the spike trains of spontaneously active striate cortex neurons. J Neurophysiol 53:926-939.

Livingstone MS, Hubel DH (1981) Effects of sleep and arousal on the processing of visual information in the cat. Nature 291:554-561.

Lu T, Liang L, Wang X (2001) Temporal and rate representations of timevarying signals in the auditory cortex of awake primates. Nat Neurosci 4:1131-1138.

Mariotti M, Formenti A, Mancia M (1989) Responses of VPL thalamic neurones to peripheral stimulation in wakefulness and sleep. Neurosci Lett 102:70-75.

Massimini M, Rosanova M, Mariotti M (2003) EEG slow (approximately 1 $\mathrm{Hz}$ ) waves are associated with nonstationarity of thalamo-cortical sensory processing in the sleeping human. J Neurophysiol 89:1205-1213.

Nielsen-Bohlman L, Knight RT, Woods DL, Woodward K (1991) Differential auditory processing continues during sleep. Electroencephalogr Clin Neurophysiol 79:281-290.

Oswald I, Taylor AM, Treisman M (1960) Discriminative responses to stimulation during human sleep. Brain 83:440-453.

Peña JL, Pérez-Perera L, Bouvier M, Velluti RA (1999) Sleep and wakefulness modulation of the neuronal firing in the auditory cortex of the guinea pig. Brain Res 816:463-470.

Pfingst BE, O'Connor TA (1981) Characteristics of neurons in auditory cortex of monkeys performing a simple auditory task. J Neurophysiol 45:16-34.

Portas CM, Krakow K, Allen P, Josephs O, Armony JL, Frith CD (2000) Auditory processing across the sleep-wake cycle: simultaneous EEG and fMRI monitoring in humans. Neuron 28:991-999. 
Poulet JF, Petersen CC (2008) Internal brain state regulates membrane potential synchrony in barrel cortex of behaving mice. Nature 454:881-885.

Rice JA (1995) Mathematical statistics and data analysis. Belmont, CA: Wadsworth.

Sadagopan S, Wang X (2008) Level invariant representation of sounds by populations of neurons in primary auditory cortex. J Neurosci 28:34153426.

Sadagopan S, Wang X (2010) Contribution of inhibition to stimulus selectivity in primary auditory cortex of awake primates. J Neurosci 30:73147325.

Steriade M (2003) The corticothalamic system in sleep. Front Biosci 8:d878-99.

Steriade M, Nuñez A, Amzica F (1993) A novel slow ( $<1 \mathrm{~Hz}$ ) oscillation of neocortical neurons in vivo: depolarizing and hyperpolarizing components. J Neurosci 13:3252-3265.

Steriade M, Timofeev I, Grenier F (2001) Natural waking and sleep states: a view from inside neocortical neurons. J Neurophysiol 85:1969-1985.
Stern EA, Kincaid AE, Wilson CJ (1997) Spontaneous subthreshold membrane potential fluctuations and action potential variability of rat corticostriatal and striatal neurons in vivo. J Neurophysiol 77:1697-1715.

Tan AY, Atencio CA, Polley DB, Merzenich MM, Schreiner CE (2007) Unbalanced synaptic inhibition can create intensity-tuned auditory cortex neurons. Neuroscience 146:449-462.

Wang X, Lu T, Snider RK, Liang L (2005) Sustained firing in auditory cortex evoked by preferred stimuli. Nature 435:341-346.

Wang XJ (2010) Neurophysiological and computational principles of cortical rhythms in cognition. Physiol Rev 90:1195-1268.

Wu GK, Li P, Tao HW, Zhang LI (2006) Nonmonotonic synaptic excitation and imbalanced inhibition underlying cortical intensity tuning. Neuron 52:705-715.

Wu GK, Arbuckle R, Liu BH, Tao HW, Zhang LI (2008) Lateral sharpening of cortical frequency tuning by approximately balanced inhibition. Neuron 58:132-143. 